\title{
Klatsch-Mohn (Papaver rhoeas): Blume des Jahres 2017
}

\author{
Armin Jagel
}

\begin{abstract}
The common poppy (Papaver rhoeas) is the flower of the year 2017. As a formerly frequent weed it has now become quite rare. It is still found along roadsides, embankments and construction sites. The common poppy has long been used as a medicinal and ornamental plant as well as a source of a coloring agent. Its biology, morphology, usage options and symbolism are presented here.
\end{abstract}

\section{Zusammenfassung}

Der Klatsch-Mohn ist Blume des Jahres 2017. Als früher häufiges Ackerunkraut ist er bei uns selten geworden. Er kommt aber noch immer an Ersatzstandorten wie Straßenrändern, Böschungen oder Baustellen vor. Der Mohn hat vielseitige Verwendungsmöglichkeiten als Farb,- Heil- oder Zierpflanze. Seine Biologie, Morphologie, Verwendungsmöglichkeiten und Symbolik werden vorgestellt.

\section{Einleitung}

Wenige Pflanzenarten sind bei uns so bekannt wie der Klatsch-Mohn. Eine knallrote Farbe wie die der Mohnblüten ist in unserer Flora selten. Denn eine leuchtend rote Blütenfarbe ist i. A. die Farbe von durch Vögel bestäubten Blumen, die es aber in Mitteleuropa nicht gibt. Die Vergänglichkeit der Blüten hat jedes Kind schon mal erfahren, das einen Strauß Mohn verschenken wollte und plötzlich nur noch blütenlose Blütenstängel in der Hand hatte. Auch die Giftigkeit der Pflanze ist wegen der offensichtlichen Verwandtschaft zum Schlaf-Mohn (Papaver somniferum) weit bekannt. Eine solch auffällige Pflanze wie der Klatsch-Mohn hinterlässt tiefe Spuren in der Sagenwelt und spielt auch in der Volksheilkunde eine Rolle.

In Deutschland ist der Klatsch-Mohn wohl der bekannteste und am weitesten verbreitete Mohn. Als so genannter Archäophyt ist er mit der Einführung des Ackerbaus als Kulturfolger aus dem Mittelmeergebiet, Eurasien oder Nordafrika nach Mitteleuropa eingewandert. Es verwundert zunächst, dass er zur Blume des Jahres 2017 gewählt wurde. Grund dafür ist, dass der Klatsch-Mohn ein klassisches so genanntes Ackerunkraut ist. Doch im Acker wächst er heute kaum noch. In diesem Lebensraum sind in Deutschland durch die Intensivierung der Landwirtschaft die meisten Artenrückgänge zu verzeichnen. Konventionell bewirtschaftete Äcker sind die wohl am stärksten überdüngten und vergifteten Lebensräume unserer Zeit. Fast alle Pflanzenarten, die sich auf

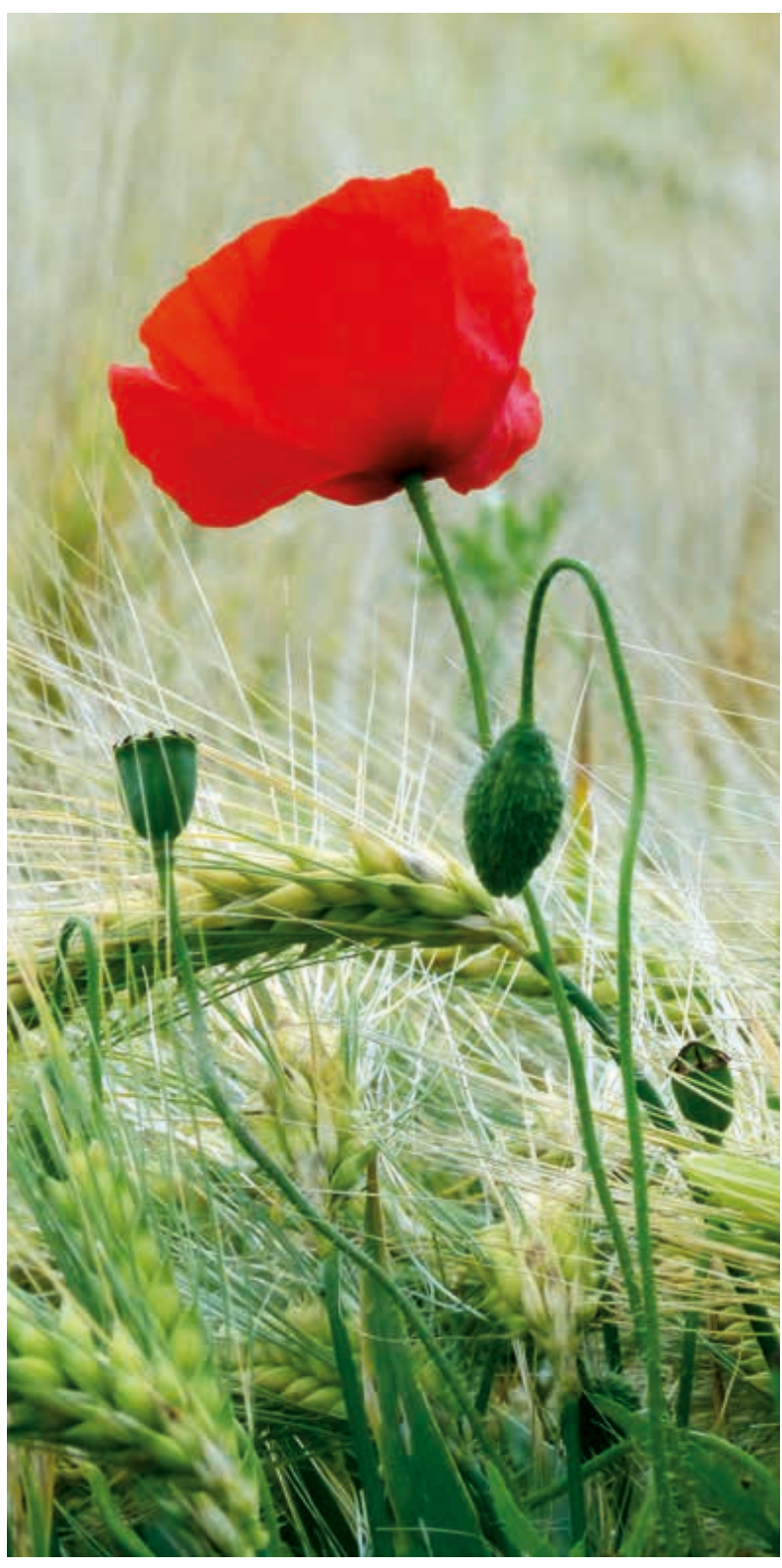

Abb. 1: Klatsch-Mohn, ein typisches Ackerwildkraut. 


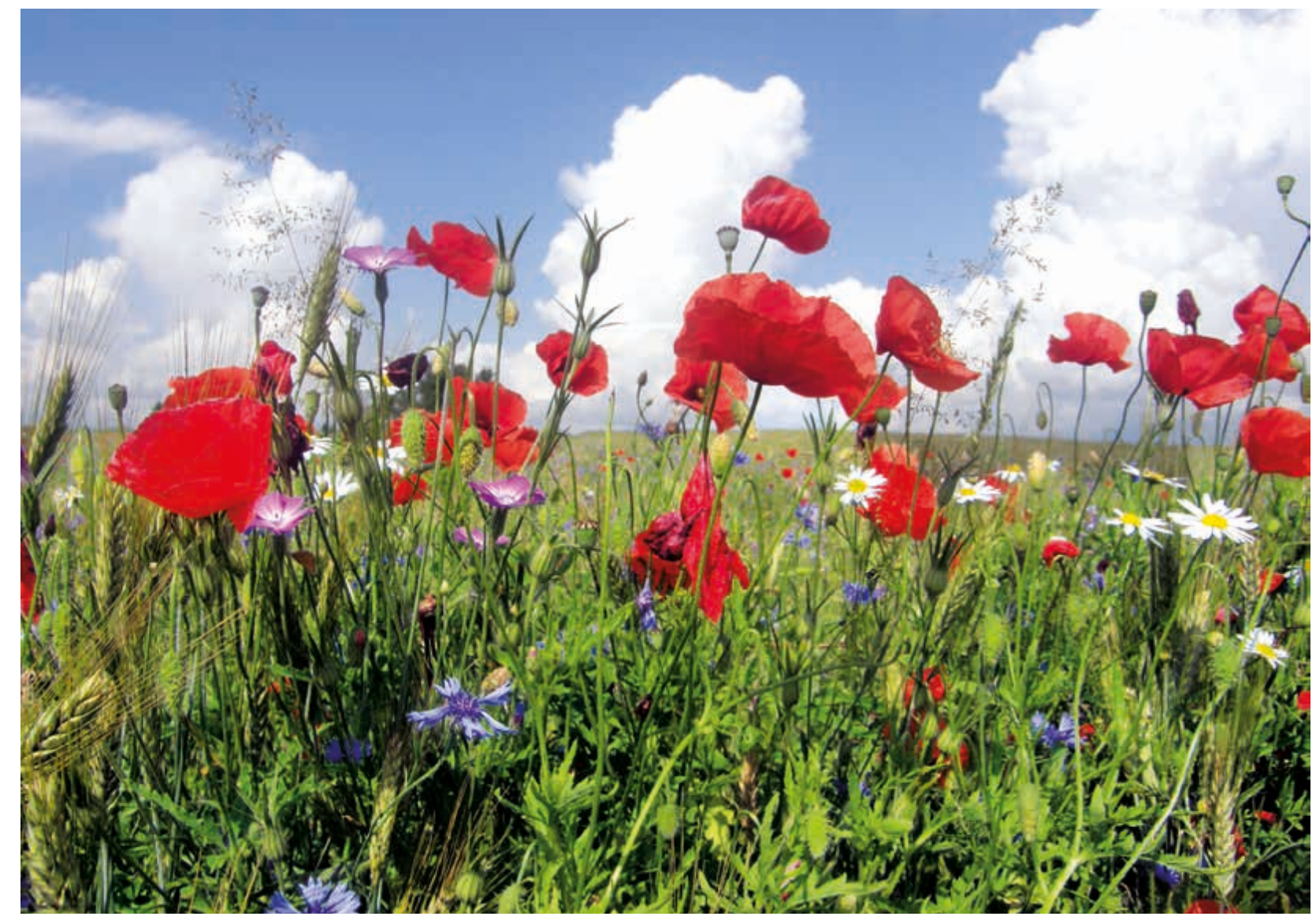

Abb. 2: Ungespritzter Acker in Masuren/Polen mit klassischen Ackerwildkräutern wie Klatsch-Mohn, Kornrade, Kornblume und Kamille.

den Lebensraum Acker spezialisiert haben, sind auf den Roten Listen der Pflanzen vertreten oder gehören darauf. Betrachtet man vor diesem Hintergrund die Bestandsentwicklung des früher sehr häufigen Klatsch-Mohns, sind die Rückgänge sehr viel massiver und offensichtlicher als die einiger heute fast ausgestorbener, aber auch früher nicht häufiger Seltenheiten, wie z. B. Acker-Haftdolde (Caucalis platycarpos) oder Acker-Lichtnelke (Silene noctiflora). Den ehemals prachtvollen Eindruck von leuchtend roten Mohnfeldern kann man heute häufiger noch in einigen Mittelmeerländern oder in Osteuropa gewinnen, bei uns ist dieser Anblick selten geworden oder häufig auf nicht gespritzte Ackerrandstreifen beschränkt. Der Klatsch-Mohn hat gegenüber vielen anderen Ackerunkräutern den Vorteil, dass Äcker nicht sein einziger Lebensraum sind. Bei uns ist er z. B. auch auf Brachland, an Straßenböschungen oder anderen ruderalen Standorten anzutreffen, wird zudem gelegentlich auch mit Sommerblumenmischungen ausgesät.

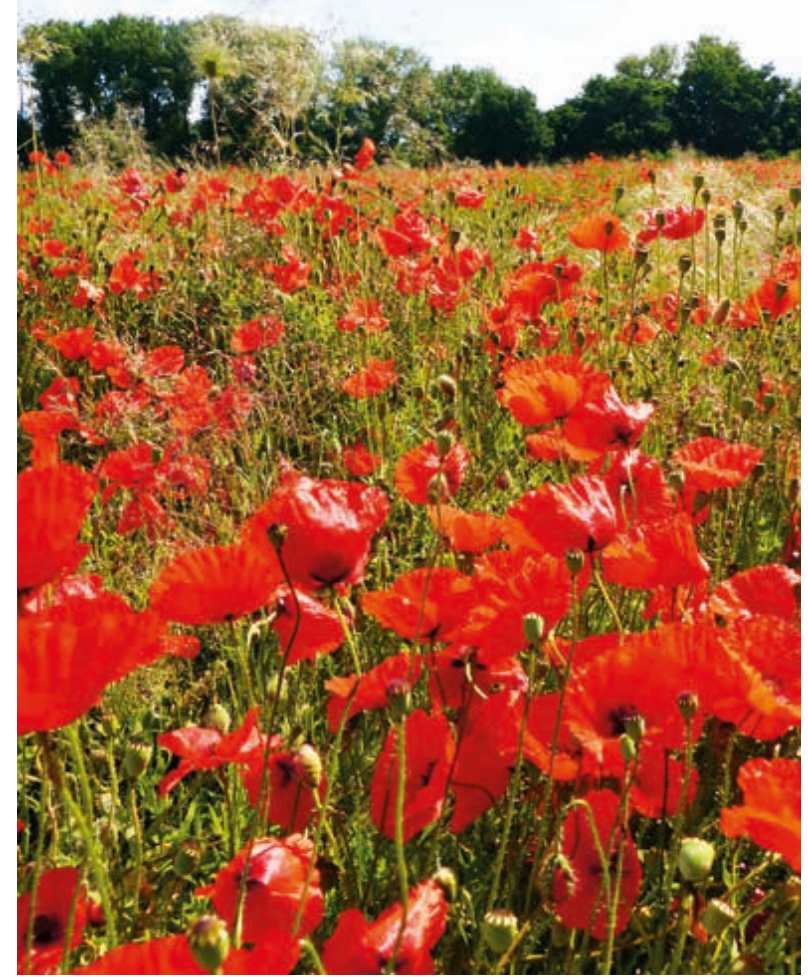

Abb. 3: Üppiger Mohnbestand auf einem Brachacker in Frankfurt (Frankfurter Berg) im Juni 2016. 


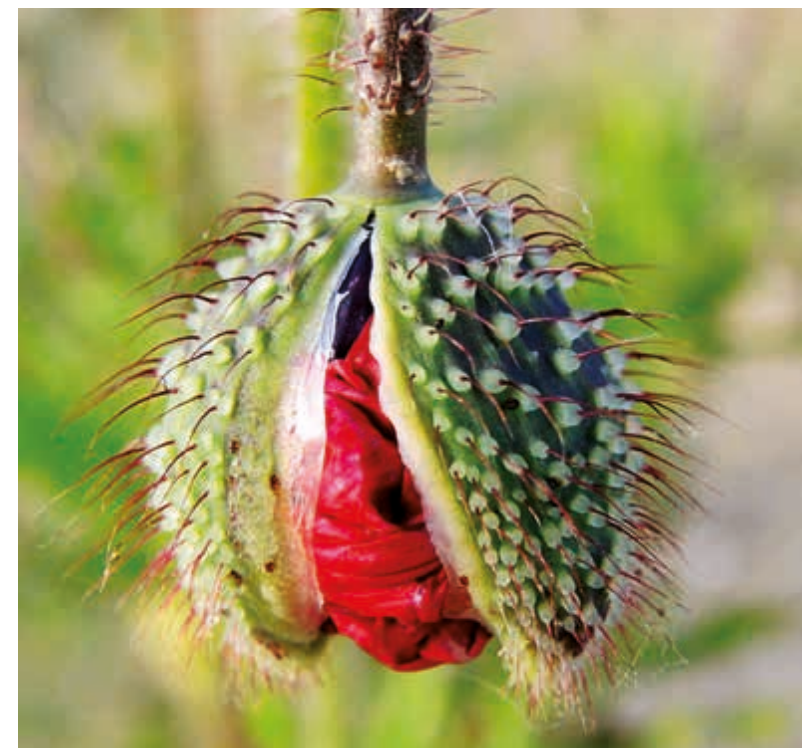

Abb. 4: Nickende Knospe eines Klatsch-Mohns.

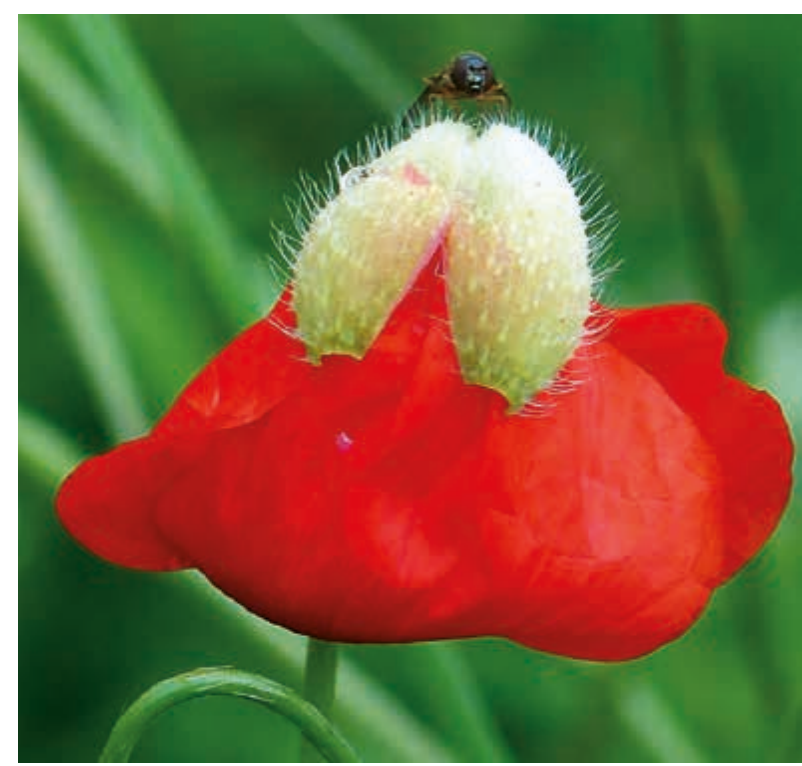

Abb. 5: Die Kelchblätter werden abgeschoben.

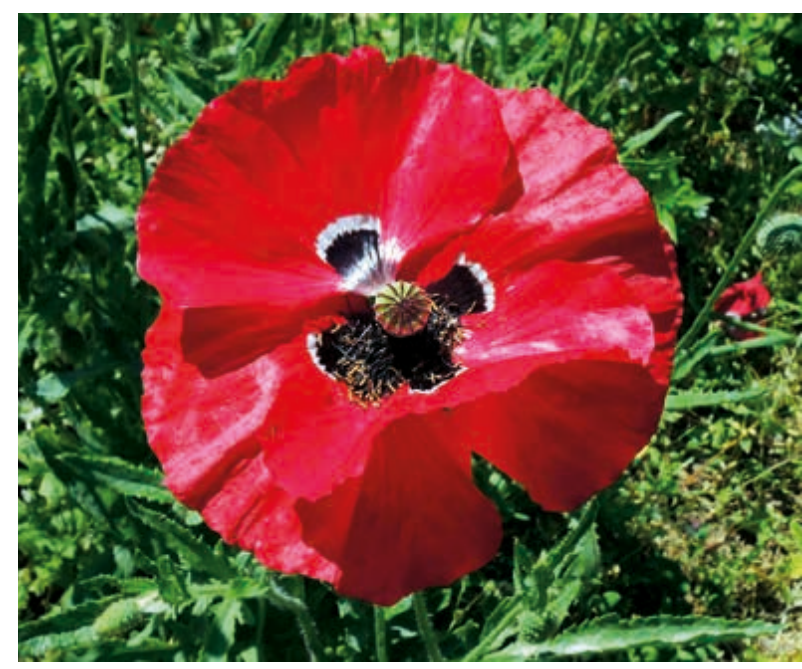

Abb. 6: Klatsch-Mohn mit dunklen Basalflecken.
Neben dem Klatsch-Mohn sind weitere Mohn-Arten in Deutschland heimisch, verwildern aus Anpflanzungen oder werden unbeständig verschleppt (vgl. hierzu Bomble \& Jagel 2016).

\section{Name}

Das Wort Mohn erscheint schon im Althochdeutschen als Mago (Magen), was sich ursprünglich auf die hohlen Kapseln des Schlaf-Mohns bezog (Beckhaus 1893, Scherf 2007). Für die Herkunft des Namensteiles „Klatsch“ gibt es viele Erklärungen. Ein beliebtes Kinderspiel war es früher, mit dem Daumen und dem Zeigefinger einen Ring zu bilden, darauf ein Blütenblatt zu legen und dann mit der anderen flachen Hand darauf zu schlagen. Dabei entstand ein klatschendes Geräusch. Für Jungen bedeutete die Lautstärke des Klatschens eine Aussage über den Erfolg bei Mädchen. Bei einem leisen Klatschen winkte ein Kuss, bei einem lauten konnte sogar auf einen weitergehenden Liebesbeweis gehofft werden (SCherf 2007). Daneben findet man häufiger die Erklärung, dass die Blüten im Wind (und bei Regen) aneinanderklatschen würden.

Das Wort Papaver stammt möglicherweise vom keltischen papa (Kinderbrei) und dem lateinischen ferre (tragen). Der Name bezieht sich darauf, dass früher schreienden Kleinkindern ein Absud von Schlaf-Mohn-Kapseln in den Brei gemischt wurde, um sie zu beruhigen. Der Artbeiname rhoeas kann verschiedenen Ursprungs sein. So ist rhoias die griechische Bezeichnung einer Mohnart. Rhoa bezieht sich auf den Granatapfel, der eine ähnliche Farbe wie Mohnblüten hat. Möglicherweise leitet sich rhoeas auch von der griechischen Vokabel rhein (wegfließen) ab, was sich auf die Milch des Mohns beziehen könnte (Düll \& Düll 2007, Düll \& Kutzelnigg 2016).

Weitere deutsche Namen des Klatsch-Mohns sind z. B. Acker-Mohn, Feld-Mohn und KornMohn sowie Blutblume, Feuer-Mohn bzw. Feuerblume (leuchtend rote Farbe), Große Klatschrose, Kornrose, Klitsche, Pfaffenblume und Klapperrose (Klappern der Samen in den reifen Kapseln). 


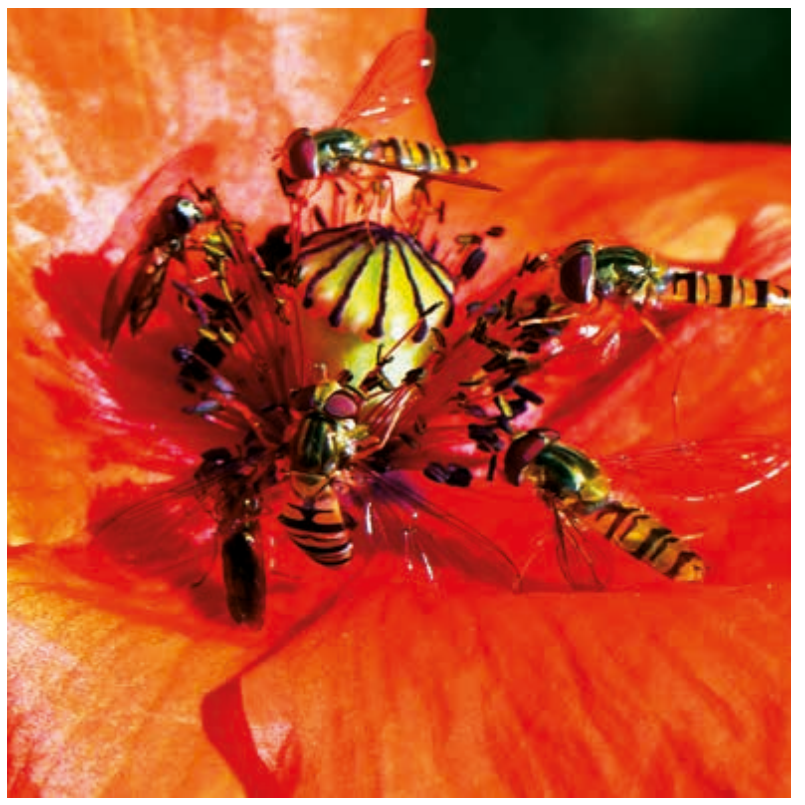

Abb. 7: Mohn wird häufig von Schwebfliegen aufgesucht.

\section{Morphologie}

Die Blütezeit des Klatsch-Mohns beginnt im Flachland in der zweiten Maihälfte und reicht bis in den September hinein. Die Knospen des Klatsch-Mohns nicken. Die zwei Kelchblätter der Mohnblüte fallen beim Öffnen der Blüte ab und geben vier anfangs noch zerknitterte Blütenblätter frei. Sie sind entweder einfarbig rot oder weisen an der Basis schwarze Flecken auf, die außerdem weiß berandet sein können.

Auch zweifarbige oder weiße Blüten kommen vor. Bei solchen Farbvariationen ist allerdings nicht immer eindeutig, ob sie aus spontanen Mutationen oder Verwilderungen aus Ansaaten stammen. Wie die Kelchblätter sind auch die Blütenblätter kurzlebig und fallen zumindest bei sonnigem Wetter und nach Bestäubung noch am Tag des Aufblühens ab. Sie sind so leicht angebracht, dass sie oft nicht einmal eine Hummel tragen können. Diese lassen sich daher oft auf der Narbenplatte nieder und bringen dabei den von einer anderen Blüte mitgebrachten Pollen gleich an die richtige Stelle, die Narben. Sollte Pollen derselben Blüte auf die Narben treffen, kommt es trotzdem nicht zur Befruchtung, weil Klatsch-Mohn selbststeril ist.

Die rote Farbe des Klatsch-Mohns ist auf Anthocyane zurückzuführen. In der heimischen Flora ist ein solch „richtiges“ Rot eine seltene Blüten-

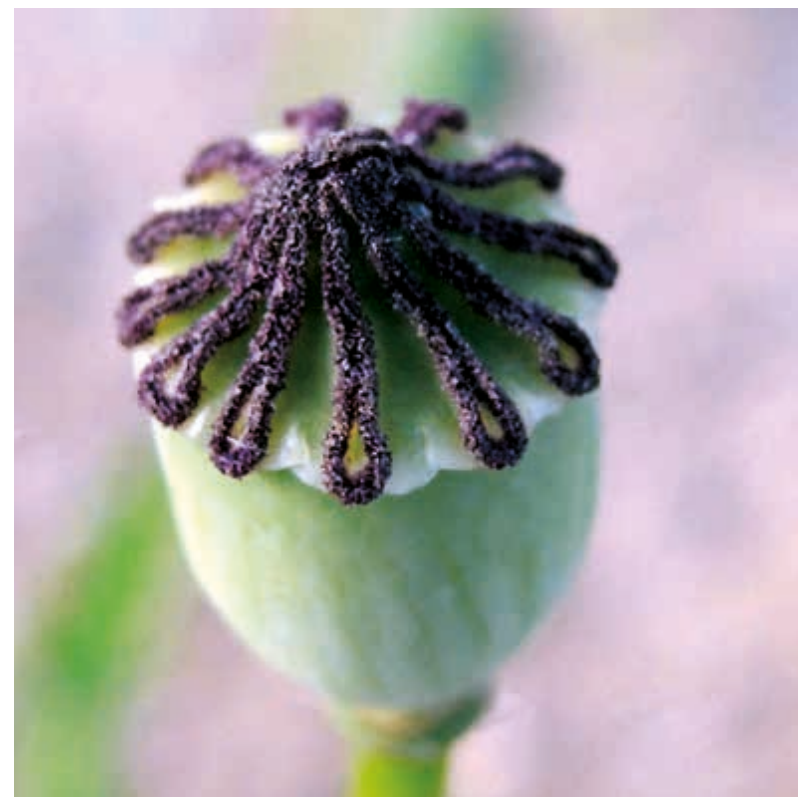

Abb. 8: Junge Fruchtkapsel.

farbe (s. o.). In der mitteleuropäischen Flora sind die meisten Blüten mit Rotanteil eher rosa, pink oder rotviolett gefärbt. Die Seltenheit von echt roten Blüten bei uns dürfte darauf beruhen, dass hier die hauptsächlichen Blütenbestäuber Bienen und Hummeln sind, die Rot nicht sehen können. Bei ihnen ist das Farbspektrum im Vergleich zum Menschen in den kurzwelligeren Bereich verschoben. Sie können allerdings Ultraviolettstrahlung wahrnehmen. Den für uns knallroten Mohn sehen Bienen nur deswegen, weil die Mohnblüten sehr stark ultraviolettes Licht reflektieren (Hess 1990, Düll \& Kutzelnigg 2016). Neben der Bestäubung durch Insekten kann es beim Klatsch-Mohn auch zur Bestäubung durch Wind kommen.

Mohnblüten duften nicht und haben keinen Nektar. Besonders zahlreich sind die Staubblätter; Mohnblüten sind so genannte Pollenblumen. Den Bestäubern, im Wesentlichen Hummeln, Honigbienen, Wildbienen und Schwebfliegen (Hintermeier \& Hintermeier 2002), bieten sie ausschließlich Pollen an, der entweder sofort gefressen oder gesammelt wird. Damit genügend Pollen zum Bestäuben anderer Mohnblüten übrig bleibt, werden gigantische Mengen an Pollenkörnern gebildet, die pro Blüte 2,6 Mio. bzw. pro Pflanze 280 Mio. erreichen können. Diese Anzahl wird von nur wenigen insektenblütigen Arten wie z. B. Pfingstrosen übertroffen (Hintermeier 

2016).

Die Früchte des Klatsch-Mohns sind eiförmige bis fast kugelige, kahle Kapseln, die sich aus zahlreichen miteinander verwachsenen Fruchtblättern aufbauen. Die Narben stehen auf einer Narbenplatte und sind als Narbenstrahlen ausgebildet, die der Anzahl der Fruchtblätter entsprechen. Direkt unterhalb dieser Platte öffnen sich zur Reifezeit Poren (Porenkapsel), aus denen die Samen entlassen werden. Der Mohnstängel ist elastisch und wird vom Wind oder von vorbeilaufenden Tieren bewegt, wodurch die Samen bis $4 \mathrm{~m}$ weit herausgeschleudert werden können (Düll \& KuTZELNIGG 2016). Der Streumechanismus der Mohnkapsel ist Vorbild für einen Salzstreuer und somit ein schönes Anschauungsbeispiel für Bionik. Die Samen sind mit etwa $0,5 \mathrm{~mm}$ Länge sehr klein, dunkelbraun und netzgrubig.

\section{Verbreitung und Vorkommen}

Der Klatsch-Mohn stammt ursprünglich wohl aus dem Mittelmeergebiet, Nordafrika oder aus Kleinasien, kommt aber seit der Jungsteinzeit auch in Mitteleuropa vor (s. o.). Heute wächst er mit Ausnahme höherer Gebirgsregionen in ganz Europa und wurde weltweit verschleppt (DüLL \& Kutzelnigg 2016).

Als typisches Ackerunkraut ist der KlatschMohn einjährig und dadurch an die Bodenbewirtschaftung angepasst, aber auch darauf angewiesen. Am häufigsten wächst er winterannuell im Wintergetreide auf kalkreichen Lehm- und Tonböden. Die Samen keimen hier bereits im Herbst und die Pflanzen überwintern als Halbrosette. Darüber hinaus kann er aber auch in Sommergetreide und seltener in Kulturen von Blattfrüchten und Klee wachsen. Dann keimt er im späten Frühjahr und ist sommerannuell. Lässt man einen Acker brachfallen, verschwindet der Mohn mit zunehmender Sukzession bald. Der gelegentlich besonders unter Fotografen verwendete Begriff Mohnwiese ist nicht richtig, denn in Wiesen kommt die Art nicht vor. Wenn man eine große Fläche mit massenhaft Mohn sieht, handelt es sich in der Regel um einen einjährigen Brachacker. Hierfür wird dann oft

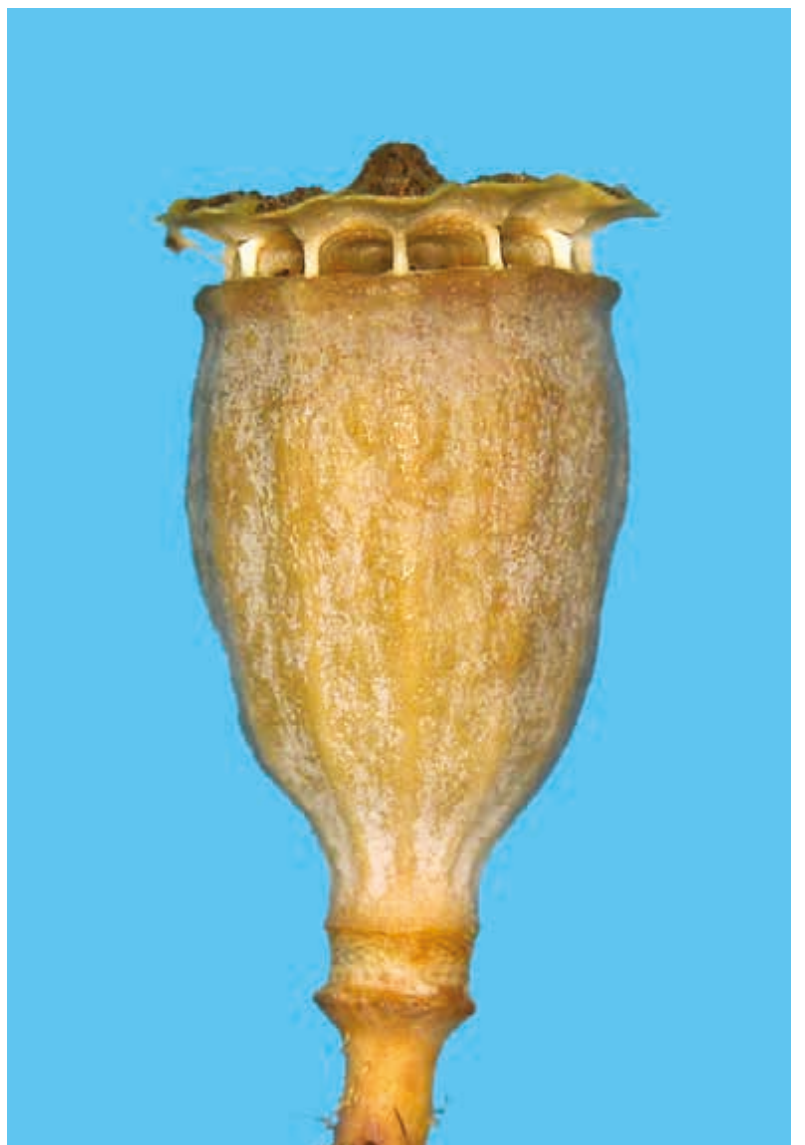

Abb. 9: Reife Kapsel, gut sichtbar die Poren.

der Begriff Mohnfeld verwendet, der sich früher mehr auf Äcker mit reichlich Mohn als Beikraut bezog. Wenn man Mohn heute in einem Acker sieht, dann fast nur noch im äußeren Ackerrand oder sogar nur noch in einem schmalen, unbestellten Streifen neben dem Acker, wo das Gift beim Spritzen nicht hingelangt ist.

Wie für viele Ackerunkräuter typisch, sammeln sich Mohnsamen im Boden an. Alljährlich keimen nur diejenigen Samen, die nach dem Pflügen flach unter der Erde zu liegen kommen (Flachkeimer). Die tiefer liegenden Samen verfaulen dabei aber nicht sofort, sondern können mindestens 10 Jahre im Boden überdauern (Cremer et al. 1991). Sie verharren dort, bis sie durch erneutes Pflügen wieder nach oben gelangen und keimen können. $\mathrm{Da}$ aber bei dieser Strategie auch eine große Menge an Samen nicht rechtzeitig an die Oberfläche gelangt, ist es sinnvoll, eine große Menge Samen zu produzieren. Beim Klatsch-Mohn sind es 20000-50 000 Samen pro Pflanze bzw. 2000 (-5 000) pro Kapsel (Cremer et al. 1991, Hanf 1990, Hintermeier 
\& Hintermeier 2002, Düll \& Kutzelnigg 2016). Da Äcker heute aber jährlich mehrfach mit Herbiziden behandelt werden, erschöpft sich der Samenvorrat mit der Zeit, bis er ganz verschwunden ist.

Aufgrund der im Boden ruhenden Samen kann es auch nach Jahren eines Brache- oder Wiesenstadiums auf einer Fläche zur Wiederbelebung des Mohns kommen, wenn eine Nutzungsänderung den Boden wieder offen legt. Hierauf beruht auch der Aberglaube von Soldaten im 1. Weltkrieg, dass auf den Schlachtfeldern blühender Mohn aus dem Blut der gefallenen Kameraden entspross (Scherf 2007).

Heute findet man den Klatsch-Mohn häufiger an offenerdigen Standorten im Siedlungsbereich, an Bahndämmen, an Baustellen und an Weg- und Straßenrändern. Diese Orte stellen keine Ersatzstandorte dar, sondern Mohn wuchs hier schon immer, nur der Stellenwert hat sich geändert, denn früher waren solche Wuchsorte gegenüber den Massenvorkommen im Acker kaum erwähnenswert.

\section{Giftigkeit}

Klatsch-Mohn ist in allen Teilen giftig durch eine Reihe von Alkaloiden, die sich besonders im weißen Milchsaft befinden. Die Giftigkeit wird dabei aber als nicht sehr stark eingestuft. Das wichtigste Alkaloid ist Rhoeadin (Rotн et al. 2012). Morphin dagegen, das den Schlaf-Mohn (Papaver somniferum) wegen der Gewinnung von Opium berühmt gemacht hat, fehlt dem Klatsch-Mohn. Von Vergiftungen wird vor allem bei Kindern berichtet, die grüne Kapseln, Blüten oder Blätter gegessen hatten und danach unter Bauchschmerzen und Erbrechen litten. Bei Pferden, Kühen und Schweinen kann es zu Krämpfen und epileptischen Anfällen kommen, wenn zu große Mengen aufgenommen werden (Rотн 2012, DülL \& KutZelnigg 2016).

\section{Verwendung}

Schon die alten Ägypter verwendeten um 1500 v. Chr. Mohnsamen wegen ihres aromatischen Geschmacks zum Backen (Hintermeier \&

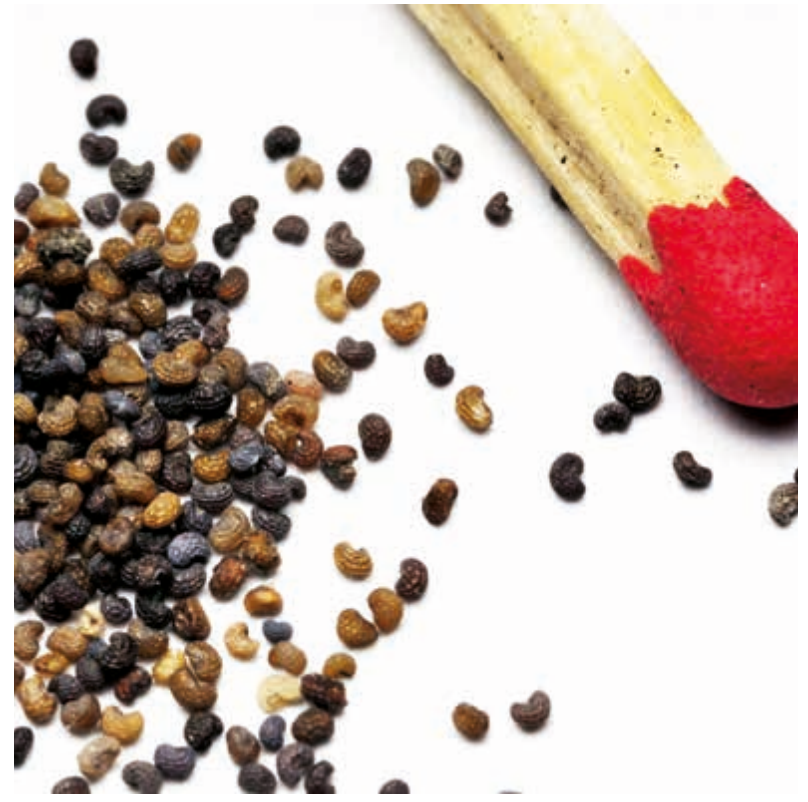

Abb. 10: Samen des Klatsch-Mohns.

Hintermeier 2002). Mohnkapseln gehörten auch zu den klassischen altägyptischen Grabbeigaben. Besonders in die Grabkammern jung verstorbener Prinzessinnen legte man Girlanden aus Mohnblüten, womöglich als Symbol des rasch verblühten Lebens (Beuchert 2004). Rote Blütenblätter wurden früher zum Färben von Wein und Sirup sowie zur Herstellung roter Tinte genutzt. Hierzu übergoss man die Blütenblätter in einem Glas mit Essig und ließ den Ansatz eine Zeitlang in der Sonne stehen (Hintermeier \& Hintermeier 2002, Scherf 2007).

Aufgrund der Wirkung der Alkaloide werden Mohne schon seit langer Zeit auch als Schlaf- und Beruhigungsmittel verwendet, wobei sie aber keine solch starke Wirkung entfalten wie der SchlafMohn (Papaver somniferum). Der Milchsaft diente dem Wundverschluss und hat antiseptische Wirkung (Düll \& Kutzelnigg 2016). In der Volksmedizin werden die Blütenblätter in Form eines Sirups gegen Husten und Unruhezustände kleiner Kinder verwendet. Der Tee wird gegen Schmerzen und als Schlaftee eingenommen (PAhlow 1983).

Aufgrund der zahlreichen Samen, der Verwendung als Schlafmittel, der Giftigkeit und der scharlachroten Farbe gilt Klatsch-Mohn als Symbol für Fruchtbarkeit, Schlaf, Vergessen, Tod, Liebesleid, Trost und die Passion Christi (Beuchert 2004). 
Da der Giftgehalt im Klatsch-Mohn insgesamt gering ist, verwendet man die jungen Blätter auch heute noch als Salatbeigabe oder gedünstet als Gemüse. Die Samen werden zum Backen genutzt und ergeben ausgepresst ein feines Öl. Die Blütenblätter werden zur Dekoration von Gerichten verwendet (FleischHauer et al. 2007). Mohnsamen auf dem Brötchen und im Mohnkuchen stammen vom Schlaf-Mohn.

Klatsch-Mohn wird schon seit dem 16. Jh. auch als Zierpflanze angeboten. In der 2. Hälfte des 19. Jahrhunderts wurde aus ihm eine Reihe von einfachen und gefüllten Sorten gezüchtet, die als Shirley-Mohn in den Handel kamen (Krausch 2007). Heute werden für die Kultur als Sommerblumen mehrere bunt blühende Sorten oder Mischungen mit oft gefüllten Blüten angeboten, die z. B. Seidenmohn (z. B. Shirley- oder Prachtmischung) oder Romantik-Mohn (Sorte 'Chorus') genannt werden. Orange-rot blüht die Sorte 'Fireball'.

\section{Dank}

Für die Bereitstellung von Fotos bedanke ich mich herzlich bei Sabine Adler (Bochum), Veit Martin Dörken (Konstanz), Detlef Mährmann (Castrop-Rauxel) und Hilke Steinecke (Frankfurt/Main).

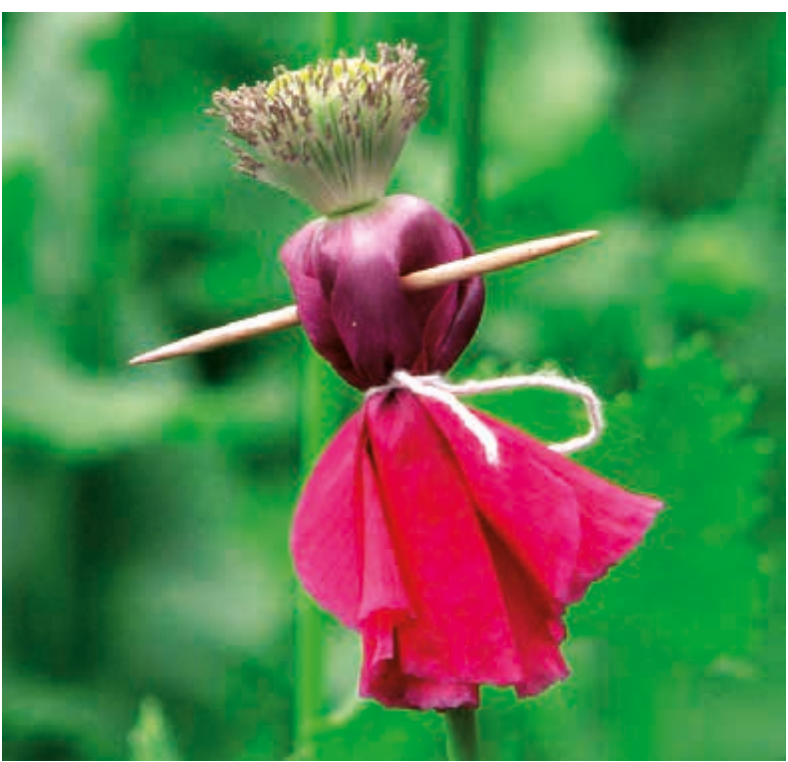

Abb. 11: Püppchen aus einer Mohnblüte.

\section{Literatur}

Beckhaus, K. 1893: Flora von Westfalen. - Münster (Nachdruck 1993).

Beuchert, M. 2004: Symbolik der Pflanzen. - Frankfurt. Bomble, F. W. \& Jagel, A. 2016: Papaver-Mohn-Arten in Nordrhein-Westfalen. - Jahrb. Bochumer Bot. Ver. 7: 237-266.

Cremer, J., Partzsch, M., Zimmermann. G., Schwër, C. \& Goltz, H. 1991: Acker- und Gartenwildkräuter. - Berlin.

DüLL, R. \& DüLL, I. 2007: Taschenlexikon der Mittelmeerflora. - Wiebelsheim.

Düll, R. \& KutzelnigG, H. 2016: Taschenlexikon der Pflanzen Deutschlands und angrenzender Länder, 7. Aufl. - Wiebelsheim.

Fleischhauer, S. G., Guthmann, J. \& Spiegelberger, R. 2007: Essbare Wildpflanzen. - Baden, München.

Hanf, M. 1990: Ackerunkräuter Europas mit ihren Keimlingen und Samen, 3. Aufl. - München, Wien, Zürich.

Hess, D. 1990: Die Blüte. Struktur, Funktion, Ökologie, Evolution. - Stuttgart.

Hintermeier, H. \& Hintermeier, M. 2002: Blütenpflanzen und ihre Gäste. - München.

Krausch, H.-D. 2007: Kaiserkron und Päonien rot. Von der Entdeckung und Einführung unserer Gartenblumen. - München.

Pahlow, M. 1993: Das große Buch der

Heilpflanzen. - München.

Roth, L., Daunderer, M. \& Kormann, K. 2012: Giftpflanzen, Pflanzengifte. - Hamburg.

Sauer, T. 1965: Unkrautfibel Schering, 6. Aufl. - Berlin. Scherf, G. 2007: Die geheimnisvolle Welt der Zauberpflanzen und Hexenkräuter. Mythos und Magie heimischer Wild- und Kulturpflanzen. - München.

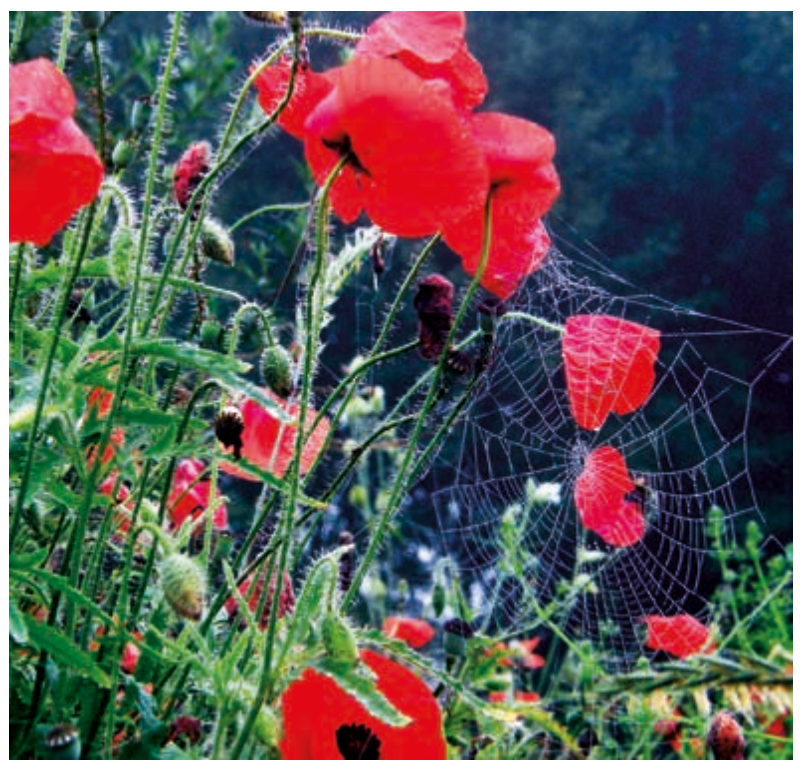

Abb. 12: Klatsch-Mohn mit Spinnennetz. 